\title{
Narrativas de reconocimiento de adultos con discapacidad: hacia una vida independiente
}

\author{
Recognition narratives for adults with disabilities: towards independent \\ living
}

\begin{abstract}
Resumen
Este texto se propone mostrar la relación entre las vivencias de reconocimiento de personas con discapacidad y su logro en la configuración de estilos de vida independiente. Se acude al método hermenéutico y al análisis bajo los postulados de la teoría fundamentada y el análisis de contenido. Se halla que el desarrollo de autoconfianza, autorrespeto y autoestima, considerados fines prácticos del reconocimiento, favorecen estilos de vida independiente en personas con discapacidad. Igualmente se encuentra que estas personas tienen experiencias denominadas enemigas del reconocimiento, que las ubica en desventaja para el logro de la autonomía y la independencia. Es importante reflexionar sobre las concepciones de discapacidad para trascender la mirada de beneficencia y facilitar la vida independiente.
\end{abstract}

\section{Palabras clave}

Vivencias de reconocimiento, vida independiente, autonomía, procesos de socialización, enemigos del reconocimiento.

\begin{abstract}
This text aims to show the relationship between the experiences of recognition of people with disabilities and their achievement in the configuration of independent lifestyles. We turn to the hermeneutic method and to the analysis under the postulates of grounded theory and content analysis. The development of self-confidence, self-respect and self-esteem as practical purposes of recognition are found to promote independent lifestyles in people with disabilities. It is also found that these people have experiences called enemies of recognition, which places them at a disadvantage in achieving autonomy and independence. It is important to reflect on the concepts of disability in order to transcend the view of charity and facilitate independent living.
\end{abstract}

\section{Keywords}

Experiences of recognition, independent life, autonomy, socialization processes, enemies of recognition.

\author{
Magda Victoria Díaz-Alzate \\ $<$ magda.diazal@amigo.edu.co>
}

Universidad Católica Luis Amigó. Colombia

\section{Natali Roldán-Berrío \\ $<$ natiroldanb@yahoo.com> \\ Alcaldía de Medellín. Colombia}

\section{Nicolasa María Durán-} Palacio

<nicolasa.duranpa@amigo.edu.co>

Universidad Católica Luis Amigó. Colombia

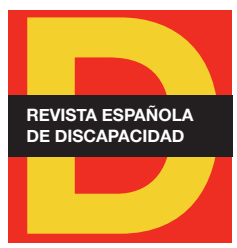

Para citar:

Díaz-Alzate, M. V. et al. (2020). "Narrativas de reconocimiento de adultos con discapacidad: hacia una vida independiente". Revista Española de Discapacidad, 8(I), pp. 63-77.

Doi: <https://doi.org/10.5569/23405104.08.01.04>

Fecha de recepción: 12-06-2019 Fecha de aceptación: 26-05-2020 


\section{Introducción ${ }^{1}$}

En este escrito se entenderá el reconocimiento desde Axel Honneth (1992, 2010), quien se centra en mostrar los aspectos morales de esta emoción, retomando la ética hegeliana, a Kant y los desarrollos sobre el cuidado de sí para alcanzar las diferentes esferas del reconocimiento (Díaz Alzate y González Bedoya, 2015: 387), y cómo ello permite el desarrollo humano para el logro de una vida digna. Se desarrolla un apartado que emerge como categoría especial para mostrar cómo algunas maneras de relacionarse con las personas en condición de discapacidad y la concepción que se tenga de esta, pueden implicar menosprecio y agravio moral, lo que se define aquí como enemigos del reconocimiento en el contexto de discapacidad.

Los desarrollos investigativos sobre discapacidad revelan que existe una tendencia a leer de manera crítica las construcciones sociales e históricas del concepto. Buitrago-Echeverri (2013: 193-216) expone la tendencia hegemónica de inscribir la discapacidad en el modelo médico-rehabilitador y propone que la salud pública debe trazarse desafíos para trascender esta mirada hacia la comprensión más política y digna para estas personas.

Por su parte, Ospina Ramírez (2010: 153-156) presenta como uno de los limitantes para el trato digno de estas personas, la negación de su abordaje desde el enfoque de derechos, y allí se halla la proliferación de actuaciones discriminatorias que impiden su reconocimiento como sujetos de derechos. Esta reflexión del autor converge con la idea de este artículo, en tanto propone que el reconocimiento de la dignidad humana es el imperativo para que haya justicia, lo cual no se desliga de la pretensión de encontrar formas para que las personas con discapacidad alcancen de manera libre un estilo de vida independiente que les favorezca desde la dignidad del ser. Reconocer esta condición en los discursos de los derechos humanos es una de las vías de construcción de justicia social y política, entendiendo que también son actores y agentes en sus desarrollos.

En esta misma vía, Hernández Ríos (2015) y Pérez y Chhabra (2019) exaltan en sus trabajos la importancia de la trayectoria sufrida por el concepto de discapacidad para hallarse en el discurso de los derechos humanos, y desde allí hacer oda a la lucha por el reconocimiento que han emprendido diferentes organizaciones y actores sociales para que se comprenda que las personas con discapacidad son sujetos plenos de derechos. Por tanto, la vida independiente como dignificante de la existencia no podría entenderse fuera de esta perspectiva.

La propuesta política pasa por comprender la experiencia del reconocimiento como emoción moral que permite alcanzar la confianza en sí mismos, develar la igualdad para demandar derechos en términos jurídicos, reconocer la pluralidad como condición humana (Arendt, 2005: 207) y la multiplicidad de cuerpos coexistiendo, en condiciones diferenciadoras. Precisamente, en los principios de la Convención sobre los Derechos de las Personas con Discapacidad, se resalta "el respeto por la diferencia y la aceptación de las personas con discapacidad como parte de la diversidad y la condición humanas” (Naciones Unidas, 2006: 5).

1. Artículo derivado de la investigación "Vivencias de reconocimiento y compasión de personas en condición de discapacidad: transitar la autonomía hacia un estilo de vida independiente", realizada en la Maestría en Intervenciones Psicosociales, avalada por la Línea de Investigación Problemáticas Psicosociales Contemporáneas de la Facultad de Psicología y Ciencias Sociales, de la Universidad Católica Luis Amigó, MedellínColombia. 
Es indudable que la concepción de discapacidad ha sufrido transformaciones en la historia. Responde a cada época y contexto. Se habla de alrededor de cinco modelos diferentes para comprenderla: tradicional o de la prescindencia, el médico, el social, el de vida independiente y el de diversidad funcional (láñez-Domínguez, 2009: 63-71). La capacidad configurativa que tiene el lenguaje para instalar prejuicios, explicaciones y acciones sobre la condición sensible de la discapacidad retrasa en algunos países el reconocimiento de estas personas como ciudadanos plenos de derechos para decidir, participar y elegir sobre sus vidas, sus cuerpos y la legislación que para ellos se construye. De allí la importancia de conocer cómo cada uno de estos modelos acuna una dinámica figurativa sobre la cual se edifican concepciones, políticas públicas y una industria económica que se vale de la discapacidad para desarrollarse.

Los modelos de la prescindencia, médico y social son los más nocivos en términos políticos. El primero concebía la discapacidad como un castigo de la divinidad, por los pecados cometidos por los padres. Las intervenciones realizadas contemplaron en Grecia y Roma, acciones eugenésicas para impedir que los niños con discapacidad crecieran, y en la Edad Media, a través de la beneficencia como caridad, pero marginándolos del resto de la población (láñez-Domínguez, 2009: 64).

El modelo médico-rehabilitador buscaba la curación de los cuerpos, lo cual implica la concepción de la discapacidad como enfermedad. Aquí el problema lo ubican en el individuo como poseedor de la deficiencia o enfermedad, lo que igualmente lo margina de la sociedad. Por último, el modelo social, que es tal vez el más utilizado en países latinoamericanos, desplaza el foco al entorno, a partir de la explicación de que es en el afuera donde se gesta la discriminación. Si bien no se puede negar la existencia del estigma social como factor que obstaculiza el desarrollo integral, investir el afuera como discapacitante es negar lo real de la limitación de los cuerpos, por consiguiente, hay una eliminación de la persona con su condición.

Es significativo rescatar que el modelo social logró un avance importante en derechos al concebir la discapacidad como un constructo social (láñez-Domínguez, 2009: 66-68), en el cual se gestan las condiciones discriminatorias y excedentarias. Este modelo nace de la tradición estadounidense respecto a la tradición anglosajona sobre los derechos civiles y la política de libertad (Carbonell, 2019: 201-202), pero se quedó relegado a la asistencia social.

Para sustentar la mirada que este artículo desarrolla, se proponen los modelos de vida independiente y de diversidad, que son considerados más cercanos a la apuesta política que aquí se defiende, en tanto otorgan estatus de sujetos políticos y ciudadanos activos.

El modelo de vida independiente nace de un movimiento de personas con discapacidad que reclaman los derechos como ciudadanos. Según Maraña (2004: 23), vida independiente se convierte en un paradigma que estipula el derecho de las personas con discapacidad a elegir en libertad sobre cada aspecto de sus vidas; esta mirada es compartida con los preceptos del modelo social. Vida independiente es un modelo político que introduce el derecho a la autonomía moral, que es diferenciada de la autonomía funcional (láñezDomínguez, 2009: 67-68) en tanto no se trata de que las personas con discapacidad hagan todo cuanto deseen, ello sería la negación de su limitación, pero sí de que puedan elegir cuál es el apoyo que requieren para cumplir con su deseo. Uno de los conceptos más importantes en este movimiento refiere a la diversidad humana: "la pretensión de igualdad surge de la concepción de la discapacidad como un elemento más de la diversidad humana [negrilla de la cita original]" (Carbonell, 2019: 203). 
El modelo de la diversidad es desarrollado por Palacios y Romañach (2006: 185-195) como una forma de trascender el modelo social y asume que los cuerpos tienen limitaciones. Para láñez-Domínguez (2009: 70) este modelo enriquece la concepción de diversidad al pasar del concepto de capacidad al de dignidad. Las personas ya no son nombradas en cuanto a la deficiencia como discapacitadas, sino como integrantes de un mundo con diversidad de cuerpos: personas con diversidad funcional. Se tuvo en cuenta el significado político de este modelo, puesto que allí se puede comprender la vivencia de reconocimiento como posibilitadora de un desarrollo humano digno, entendiendo que la atención a la discapacidad ya no puede ser reducida al otorgamiento del derecho a la vida, sino que debe trascender su propósito a los derechos humanos más amplios.

Estos modelos confluyen en la importancia de saberse diversos, lo que favorece el proceso del reconocimiento en los diferentes ámbitos de participación. Para láñez-Domínguez: "el respeto de la diversidad es el principio del camino para lograr la igualdad" (2009: 71), y ello deviene de la posibilidad de vivir lo que Honneth (1996: 14-15; 2010: 21-35) nombra como las tres formas básicas del reconocimiento, siguiendo a Hegel y sus primeros desarrollos teóricos sobre la eticidad (Díaz Alzate y González Bedoya, 2015: 80). La primera forma, relacionada con el amor y la valoración de la persona por parte de los seres afectivamente más cercanos, se despliega para desarrollar la autoconfianza. La segunda forma requiere la ampliación del espectro relacional más allá de los lazos afectivos cercanos para el encuentro con otros seres humanos iguales, como especie y en la esfera jurídica, lo cual implica el reconocimiento del otro en la igualdad de derechos.

La tercera forma, ya no sólo deviene en reconocimiento de igualdad; aquí está la implicación de lo que reconoce Arendt (2005: 207) como pluralidad en la humanidad, y es la condición de saberse iguales y a la vez diferenciados. Para Honneth (2010: 29) esta última forma tiene como fin el desarrollo de la autoestima, y el proceso implica la empatía solidaria del relacionamiento ético entre los humanos, reconocidos como individualizados.

Estas formas las desarrolla Honneth (2010: 23-29) con relación a las antagonistas formas de desprecio o menosprecio: maltrato, desposesión de derechos y degradación del ser, que es la manera negativista de entender los daños morales en las relaciones humanas, lo que implica, según Fernández y Vasco (2012: 471), una desposesión de derechos que puede llegar a anular el respeto por sí mismo y, en esa medida, a la no consideración propia como personas legítimas para la interacción. Por tanto, para la propuesta de este artículo, la vivencia de las formas de reconocimiento no solo favorece la capacidad para desarrollar autoconfianza en primera instancia, sino que contribuye de manera significativa al reconocimiento de sí mismo como persona legítima de derechos para tomar decisiones sobre su vida, con pleno conocimiento de las limitaciones de los cuerpos, pero entendiendo que ello hace parte de la condición humana de la diversidad.

\section{Metodología}

Para alcanzar el objetivo propuesto de comprender las vivencias de reconocimiento en diferentes ámbitos de participación de personas en condición de discapacidad, que favorecen la autonomía y la configuración 
de un estilo de vida independiente, se selecciona la investigación cualitativa. Este tipo de investigación sitúa al sujeto participante como actor de su realidad (Galeano, 2004: 19-24), como aquél que mejor la conoce, la siente y la puede narrar. Para Strauss y Corbin (2002: 20), la investigación cualitativa trata de las vivencias, comportamientos, emociones, acciones, que son aspectos no susceptibles de ser tratados de manera meramente estadística.

Se halla en el método hermenéutico la posibilidad de alcanzar el objetivo descrito. Para Martínez Miguélez (2004), la hermenéutica permite develar significados de aquellas vivencias narradas por tratarse de un método que comprende al sujeto en su historicidad y discurso. Como técnica de recolección de información, se utilizó la entrevista cualitativa o en profundidad (Valles, 2002) para dar libertad a la narración de los participantes según los significados construidos por ellos.

Para la selección de los participantes se consideraron criterios de inclusión que además sirvieron como datos para la caracterización de la población informante. El primer criterio fue de orden ético. Se aplicó la Escala de Intensidad de Apoyos (en inglés Support Intensity Scale - SIS) (Thompson et al, 2004) con el propósito de definir la capacidad para tomar la decisión de participar de manera autónoma, siendo este uno de los criterios fundamentales para la selección. Asimismo, se definieron criterios de condición y edad: personas entre 30 y 40 años, con discapacidad física, visual, cognitiva o múltiple, denominación según la Clasificación Internacional del Funcionamiento (CIF) (Organización Mundial de la Salud (OMS), 2001). Respecto a las condiciones socioeconómicas, hubo coincidencia en que todos los participantes pertenecen a la clase económica baja según la clasificación colombiana, de acuerdo con el nivel de ingresos económicos y la capacidad adquisitiva. Asimismo, la presencia de la discapacidad debía ser de nacimiento o adquirida en la primera infancia. A continuación, se describen los participantes, que fueron seleccionados con el criterio de conveniencia, por la facilidad de acceso y el interés demostrado para estar en la investigación:

- $\quad$ PCDF: Mujer. 40 años. Discapacidad física desde el nacimiento. Prestadora de servicios de la Alcaldía de Medellín, Colombia. Vendedora de productos por catálogo y deportista. Vive con su hermana.

- $\quad$ PCDV: Hombre. 31 años. Discapacidad visual desde el nacimiento. Profesional en planeación y desarrollo local de la Universidad Pascual Bravo de Medellín, Colombia. Vive solo.

- $\quad$ PCDM: Mujer. 38 años. Discapacidad múltiple (mental y física) desde el nacimiento. Estudiante de lengua de señas colombiana. Integrante del Comité Comunal de Inclusión de la Comuna 12, Medellín, Colombia. Vive con su hermana.

- $\quad$ PCDC: Hombre. 38 años. Discapacidad cognitiva. Técnico en fotografía y bailarín profesional. Vive con sus padres y un hermano.

Para registrar la información se utilizó la grabación con consentimiento de los participantes. Este material de audio se transcribe para facilitar el acceso a los datos orales y tener la posibilidad de volver a leer el texto. Se utilizó un cuaderno de notas para registrar expresiones, ocurrencias, primeros análisis de relaciones en las entrevistas y las observaciones que no pudieron ser captadas por la grabadora.

Aquí el análisis de la información se concibe como un tejido entre los saberes y experiencias narradas por los participantes y la interpretación. Este proceso se realizó con base en las propuestas de Coffey y Atkinson (2003) y de Strauss y Corbin (2002), de manera que la codificación y categorización trascendieran la des- 
cripción para la construcción de sentidos más profundos. Fue necesaria la utilización de análisis de contenido como herramienta metodológica secundaria (Ruiz Silva, 2004), para organizar la información transcrita y profundizar en las expresiones más recurrentes de los participantes.

La validación de los hallazgos se realizó a través de la triangulación en dos tiempos. En un primer momento, se presentan las interpretaciones a los participantes, quienes las validaron. Este momento es la triangulación con la fuente primaria. En un segundo momento, se realiza contrastación documental (Martínez Miguélez, 2004: 276-278) con los antecedentes y los referentes teóricos, y un intercambio con investigadores de la Línea de Investigación Problemáticas Psicosociales Contemporáneas de la Universidad Católica Luis Amigó, que es la triangulación con pares académicos. Es importante mencionar que se utilizaron herramientas tecnológicas como ayudas para la organización de la información: ATLAS.ti versión 8 y matrices analíticas.

Se contó con el consentimiento informado como documento legal de responsabilidad con los datos y la información de los participantes. Durante la firma de este documento se explicaron los objetivos de la investigación, el interés científico y académico de la misma, y el compromiso de salvaguardar la identidad y otros datos personales.

\section{Resultados}

La investigación de la cual se deriva este artículo tuvo como resultados dos supracategorías: (1) Vivencias de reconocimiento y compasión en ámbitos de socialización y participación, y (2) Experiencias de reconocimiento y compasión, que favorecen la autonomía y configuran estilos de vida independiente. Esta última contiene la categoría que aquí se presenta y que pretende describir la relación entre las experiencias de reconocimiento que viven las personas con discapacidad y el desarrollo de estilos de vida independiente. También se halló lo que se nombra como enemigos del reconocimiento en la discapacidad como sentido emergente manifiesto en las trayectorias de vida de las personas entrevistadas, que se han presentado como obstáculos para su desarrollo en momentos específicos.

\subsection{El reconocimiento y los estilos de vida independiente}

Las narraciones de los participantes respecto a sus experiencias de socialización en diferentes espacios permitieron develar características del reconocimiento conceptualizado por Honneth $(1992,2010)$ desde una perspectiva ética, lo que se relacionó con la posibilidad de aparición en el espacio público de las personas en condición de discapacidad con cuerpos diversos y con las propias limitaciones. Ello no solo favoreció el desarrollo de habilidades relacionales, sino que también reconfiguró el significado que tienen sobre sus innegables limitaciones, lo que permite la configuración de un estilo de vida independiente, si se comprende la independencia relacionada con la autonomía moral (láñez-Domínguez, 2009).

Para Honneth (1997), el devenir del sujeto está relacionado con el saberse reconocido por otro desde sus propias facultades y capacidades, lo que permite la construcción de una imagen de sí mismo en sentido 
de valía y confianza para la aparición social. Los participantes dan cuenta de las experiencias de reconocimiento como acontecimientos en los cuales las relaciones de empatía y valoración del otro sirvieron de correlato para la configuración de la legitimidad como personas capaces de tomar decisiones. Aquí subyace la relación entre las vivencias de reconocimiento y la configuración de estilos de vida independiente, en tanto desarrolla las capacidades para tomar decisiones a partir de la autoconfianza (esfera del amor), el autorrespeto (esfera de la ley) y la autoestima (esfera del logro) (Honneth, 2004), y se permite el despliegue de la autonomía moral, que está directamente ligada a la construcción de las condiciones para alcanzar la independencia. A continuación, se presentan los hallazgos de esta supracategoría, de acuerdo a las tres formas de reconocimiento:

\subsubsection{Primera forma de reconocimiento. El logro de la autoconfianza}

Según Honneth $(1996,2010)$, la primera forma de reconocimiento se circunscribe en las relaciones más íntimas que no se reducen a la familia. No se trata de la relación por necesidad biológica. Se refiere en un sentido amplio a la motivación para encarar la necesidad humana de existencia del otro, que solo es posible en la medida en que otro humano otorgue ese lugar concreto. Siguiendo a Mead (1968), el yo, como resultado de la autorreferencia, solo es posible en la relación con otros, puesto que, cuando se le otorga un lugar moral a ese ser, él puede reconocerse concreto:

"El hecho de que el ente hombre puede estimularse y ante su estímulo puede reaccionar como ante los estímulos de los otros, dispone en su comportamiento la forma de un objeto social, del que puede brotar un 'yo' al que pueden referirse las experiencias denominadas subjetivas" (Mead, 1968: 225).

Para PCDF, la persona que encarna esta experiencia de acogida es la abuela, quien le otorga un lugar concreto en el mundo social a partir de la enseñanza de quehaceres cotidianos en el hogar. Esta actuación se da en la socialización primaria que para Berger y Luckmann (2001) es el proceso que introduce a las personas a la sociedad y que no está únicamente a cargo de los padres, también de cualquier persona que haga las veces de agente socializador. Aquí deviene una persona con confianza en sí misma, como parte del proceso de desarrollo humano. Este tipo de confianza es posible si el niño halla que su cuidador tiene confianza en él, tanta, como para dejarle sin supervisión mientras realiza actividades (Erikson, 1985). Dice PCDF:

"Ella me enseñaba cómo cuidar a los animales, me enseñaba cómo se cosía, cómo se cocinaba y todo; de hecho, yo aprendí a cocinar por ella. Ella me enseñó a cocinar, me enseñó a coser. Ella misma me hacía la ropa y me enseñaba cómo se hacía, o sea, toda la parte del hogar lo aprendí por ella" (2 de septiembre de 2017).

Este relato contiene dos sentidos: el primero, aborda la relación de confianza que la abuela otorga a PCDF respecto a las capacidades para la ejecución de tareas de hogar, y el segundo apunta a la formación en autonomía sobre las acciones de la cotidianidad. La importancia está en que la abuela logra trascender la mirada de beneficencia para reconocer en PCDF capacidades que pueden menguar la dependencia en un futuro:

"Mi abuela me enseñó a ser muy independiente, me enseñó a que fuera capaz de hacer todas las cosas, de que yo me enfrentara a todo, ella me enseñó desde cómo se lavaba, cómo se cocinaba, cómo se hacía todo lo de casa para 
que yo aprendiera y no tuviera que depender de nadie, para que yo misma aprendiera a hacer mis cosas, fuera útil sin necesidad de depender de nadie" (PCDF, 2 de septiembre de 2017).

PDCV coincide en la importancia que tiene para el desarrollo de la autonomía la posibilidad de ser y de hacer que otorga la familia en los primeros años cuando existe una discapacidad:

"Yo creo que hay dos conceptos: la autonomía y la funcionalidad, porque si ellos me lo hubieran hecho todo y no me dejaran hacer nada, con toda seguridad yo no sería el mismo. Sería una persona con discapacidad sobreprotegida y sin trabajo" (2 de septiembre de 2017).

Este proceso formativo en la socialización primaria se relaciona con la primera esfera de reconocimiento y el fin de este proceso es la autoconfianza, lo que favorece en adelante el desarrollo de autonomía, en tanto construye capacidad para tomar decisiones básicas en las tareas cotidianas, y es posible, por la implicación afectiva de quienes cuidan y el reconocimiento de las capacidades para el despliegue de esa autonomía.

\subsubsection{Segunda forma de reconocimiento. El logro del autorrespeto}

En la segunda forma del reconocimiento, la reciprocidad coincide con la renuncia egocéntrica de los primeros años del ser humano. Esta posibilidad de ampliación de las relaciones, más allá de los íntimos, se comienza a gestar en los procesos de socialización secundarios (Berger y Luckmann, 2001), con el ingreso a otras instituciones diferentes a la familia y cuando se presentan otros que son humanos también, ciudadanos y parte del mundo de la vida más expandido. En esta esfera o forma que Honneth $(1996,2010)$ denomina de la ley, se alcanza el autorrespeto. Ello es posible en la relación recíproca simétrica, en la cual hay una igualdad de derechos imprescindible para la valoración mutua. El autorrespeto se construye por saberse igual a otro, con lugar en el mundo como el otro: "reconociendo a alguien en el sentido de concederle una autoridad moral sobre mí, estoy motivado al mismo tiempo a tratarlo en el futuro de acuerdo con su valor" (Honneth, 2011: 176).

La expansión de la experiencia de socialización con otros no íntimos en la discapacidad tiene una connotación política porque las sociedades son las que favorecen o no este proceso, de acuerdo a la concepción que tengan de la misma condición. Aquí reside la importancia de conocer los diferentes modelos sobre la discapacidad, para que haya una concordancia entre lo que se concibe y las decisiones políticas que se toman para favorecer esta población o eliminarla como sujetos de derechos. Esta esfera es vivida en espacios cotidianos y en la posibilidad de socializar con personas en su misma condición.

Para PCDM, el contacto con personas ajenas a su familia le permitió reconocer su limitación para relacionarse y utilizar el lenguaje hablado, en tanto la enfrentaba a la mirada de otros que desconocían sus diagnósticos. El darse cuenta le permite avances en su socialización. Para el proceso de configurar un estilo de vida independiente es tan importante el reconocimiento de aquello que favorece el desarrollo humano, como de aquello que limita la expansión de la subjetividad:

"Ellos me acompañaban [refiriéndose a su familia], me sacaban a la calle, a caminar, a ir a fiestas, a ir a reuniones, a hablar con los demás, a sentir contacto entre (sic) las personas y ya superé eso, ya hablo común y corriente con las personas, ya las miro casi de frente, no agacho mucho la cabeza" (PCDM, 22 de septiembre de 2017). 
Estas experiencias no solo fortalecen la confianza en sí, sino que además ubica a los participantes en relaciones simétricas, es decir, provocan el encuentro cara a cara con otro sujeto en igual condición de humanidad. Para PCDF el encuentro con otros sujetos que comparten el hecho de devenir humano y la condición de tener algún tipo de limitación, favoreció el alcance de autonomía:

"Cuando estaba estudiando en el ITM², a mí me llevaban y me traían como una muchachita, como un muñequito, ahí me descargaban, ahí me recogían, pero ya empezar a socializar con otras personas con discapacidad, entonces aprendí a rodar (sic), cosa que yo no hacía porque era muy miedosa, entonces ya aprendí con ellos a salir a rodar, a conocer otros sitios, cómo irme, otros transportes, sin tener que estar a toda hora pendiente del mismo taxi. Hasta aprendí a montar en Metroplús y buses. Eso es un proceso muy importante porque aprendí a relacionarme con otras personas con discapacidad, pero también aprendí a conocer otras cosas que yo no conocía" (PCDF, 2 de septiembre de 2017).

Dichas vivencias son posibles siempre que las sociedades constituyan un modelo de discapacidad que reconozca la vida digna como derecho de los ciudadanos, independientemente de su condición o capacidad de aporte productivo. Para Nussbaum (2012) el autorrespeto es posible en la medida en que las sociedades realmente se orienten por el camino de la justicia, en tanto responda con la protección de la dignidad de sus ciudadanos, de tal manera que la igualdad cobre sentido, no como condición homogeneizadora, sino también en calidad de garantía de derechos para el desarrollo humano.

\subsubsection{Tercera forma de reconocimiento. El logro de la autoestima}

La simetría no puede ser entendida como la excusa para homogenizar las vidas humanas: “'simétrico' debe significar que cada sujeto obtiene sin escalonamientos colectivos la oportunidad de experimentarse en sus obras y capacidades propias como valioso para la sociedad" (Honneth, 2009: 304). Aquí la tercera forma del reconocimiento se hace posible. La solidaridad permite alcanzar la autoestima, que es el fin práctico de esta vivencia de reconocimiento:

"la solidaridad depende de la condición previa de que existan relaciones sociales de valoración simétrica entre sujetos individualizados (y autónomos); en este sentido, valorarse simétricamente significa mirarse mutuamente a la luz de los valores que hacen aparecer las capacidades y propiedades del otro como significativas para la praxis común" (Honneth, 2009: 304).

En los participantes esta esfera está definida por las oportunidades escolares, laborales y de vivencia de otros espacios de socialización y desarrollo:

"Más o menos en el 2013, me aparece la primera oportunidad de trabajar, como ser un poco más independiente. Comencé a trabajar en un proyecto de la Alcaldía de Medellín en el Parque Juanes. Al tener una parte "económica" más estable, empecé a estudiar, empecé a trabajar y estudiar. En ese tiempo me sirvió para que pudiera terminar la técnica, a hacer las prácticas; hacerme ya a mis cosas, ir pagando una cosa, la otra, el portátil, celular, y a ir viendo otras capacidades que yo tenía y que podía hacer muchas cosas, que podía trabajar, estudiar y hacer mi vida, común y

2. El Instituto Tecnológico Metropolitano (ITM) es una Institución de Educación Superior pública de la ciudad de Medellín, Colombia, que ofrece formación tecnológica, profesional y posgradual en especializaciones y maestrías. 
corriente, como cualquier otra persona. Ahí fue donde yo vi que sí, que uno sí es capaz, que simplemente le falta un empujoncito o una oportunidad de uno poder como abrirse más, conocer más" (PCDF, 2 de septiembre de 2017).

Para Honneth (2010) en esta esfera se da el reconocimiento de exhortación recíproca. Las personas son apreciadas en su individualidad, de acuerdo a las capacidades para aparecer en la sociedad: "será finalmente reconocida como una persona aquella cuyas facultades sean de un valor constitutivo para una comunidad concreta" (Honneth, 1996: 14). Ello se puede leer en el relato de PCDC:

"Ya con lo de la entrevista me han conocido mucho más (sic), fuera de eso grabé como unos 12 programas con la Viejoteca de Telemedellin ${ }^{3}$. He conocido muchos artistas muy buenos, mucha gente me vio; vio cómo bailaba, la técnica que tengo, entonces me ha ayudado mucho también. Fuera de eso, he participado con otro amigo mío coleccionista, con dos libros, el primero era sobre la vida y obra de Lucho Bermúdez, toda la vida de él desde que empezó en lo de la música, hasta su final de su vida, ahí estuvo mi aporte también, ese libro se ha vendido muy bien a nivel del país y en el mundo" (PCDC, 7 de octubre de 2017).

La relación recíproca de mirada sobre la dignidad humana solamente es posible si se tiene como principio que el otro es un fin en sí mismo, como bien lo enuncia Nussbaum (2012), siguiendo las ideas de Kant. Es posible una sociedad justa, si el otro aparece con rostro concreto, con capacidades y limitaciones, reconocido como un ser humano que deviene en igualdad de derechos y diferenciado por el proceso de configuración subjetiva.

\subsection{Enemigos del reconocimiento en el contexto de la discapacidad}

El concepto de reconocimiento propuesto por Honneth (2010) está basado en un enfoque negativista de daños morales que se contrapone a las tres esferas descritas. El primer daño moral habla del maltrato físico que se recibe de los más cercanos, donde el sentimiento de agravio no lo suscita el dolor físico, sino conocer la intención de maltratar que tiene el otro en el que se confía. Es importante resaltar que los participantes de esta investigación no relataron ninguna experiencia respecto al primer daño moral.

En segundo lugar, está la negación de derechos, que es uno de los agravios morales más frecuentes para las personas con discapacidad. El menosprecio es evidente cuando hay negación de la diferenciación de los cuerpos, puesto que hay un solapamiento de los Estados en equiparar a todos como iguales, y así no actuar de forma equitativa respecto a los niveles de apoyos requeridos según las diversidades. Para Nussbaum (2012) una de las limitaciones para que haya justicia social es pensar que todos los seres humanos están en igualdad de condiciones en términos de libertad e independencia. Esta creencia ha obstaculizado la garantía de derechos como la educación y la asistencia, puesto que niegan la existencia de las limitaciones de la discapacidad y no evidencian las actuaciones de injusticia de Estados inequitativos.

Sobre el tercer daño moral, en contraposición a la relación solidaria, Honneth (2010) ubica el agravio en la degradación del valor social. En las sociedades estructuradas desde las relaciones jerárquicas, con valoraciones a partir de la capacidad de producción, la apreciación de los individuos se reduce al cumplimiento de los preceptos establecidos por un orden social cívico, descuidando la valía por el hecho mismo de ser hu-

3. Telemedellín es el canal de televisión de la ciudad de Medellín, Colombia. 
mano: "la estima social adopta con este proceso de individualización un patrón que se otorga a las formas de reconocimiento ligadas a ella el carácter de relaciones asimétricas entre sujetos individuados en términos de su historia de vida" (Honneth, 2009: 330).

En estas formas de menosprecio se ubican los enemigos del reconocimiento en la condición de discapacidad. PCDF sobre la relación con algunos integrantes de su familia, refiere:

\footnotetext{
"Yo soy muy distante de ellos porque me crie muy aparte, porque, como le digo, yo soy la única persona - de doce hermanos que éramos - , con discapacidad, entonces desde los dos meses a mí me dejaron... me tocó quedarme en un hospital mientras mi mamá se iba y cuidaba a los otros hermanos míos, entonces casi siempre yo me crie muy distante de ellos. Eso hace que yo sea muy apática de (sic) ellos, o sea, no apática de ser mala clase con ellos, sino que sea muy desprendida de ellos" (PCDF, 2 de septiembre de 2017).
}

La concepción de la discapacidad como enfermedad y la inoperancia de políticas públicas en Colombia que favorezcan el acompañamiento de estas personas hacen que su desarrollo humano tenga obstáculos que dificultan la configuración de vidas independientes. Para Nussbaum (2012), por ejemplo, existen dos problemas en la asistencia con justicia social a personas con discapacidad. El primero está ligado a la pregunta por el trato justo a unas personas que sin duda requieren apoyos específicos para su desarrollo digno y alcanzar niveles de autonomía para la configuración de vidas independientes. El segundo problema lo ubica en las condiciones de los cuidadores, a quienes no se les reconoce su labor y terminan teniendo también limitaciones para tener una vida libre, con la particularidad, además, de confinar a las mujeres a este encargo de cuidado.

Además, la actitud de los padres frente a las condiciones de limitación de sus hijos fue percibida como obstáculos iniciales en sus desarrollos. Aquí se muestran dos extremos, el primero trata de la negación de la limitación y la exigencia en la ejecución de tareas:

Él quería que yo cogiera las cosas como él las cogía y no, porque yo de todas maneras tenía mi deficiencia y él quería las cosas rápidas y no, yo me sentía mal y siempre lo hacía a espaldas de mi mamá, que mi mamá no se fuera dar cuenta, porque ya sí tenían el problema, él fue muy asolapado (sic) en ese sentido. (PCDC, 7 de octubre de 2017).

El segundo extremo trata de la actitud de sobreprotección, lo que limita el desarrollo de la autonomía y exacerba la dependencia: "mi papá siempre era el rechazo a que yo estuviera con niños que no tuvieran discapacidad, que porque me iban a maltratar, que porque qué pesar de mí, que yo con discapacidad cómo iba a estudiar allá" (PCDV, 2 de septiembre de 2017).

Estas relaciones degradan el ser moral, lo desconocen en sus particularidades y niegan las capacidades para tomar decisiones. Aquí hay una suerte de imputabilidad moral, según Honneth (2010), pues se reduce a los individuos a una expectativa de ser humano creada por sociedades higienistas, modelos de cuerpos que se imponen (láñez-Domínguez, 2009) y que, incluso, sustentan las políticas de inclusión escolar, que no siempre favorecen el desarrollo humano digno:

"Después de que quedé ciego totalmente, ahí ya sí empezó una etapa de encierro y hubo algo que me afectó mucho, que fue la Ley de integración escolar, que fue la Ley 762 y la Ley 361, que fue la Ley de inclusión. Había confusiones 
en el Estado sobre lo que era inclusión y lo que era integración. En ese orden de ideas tuve que salir de muchas escuelas, porque se decía que los niños debíamos pasar a escuelas regulares, ¿sí?” (PCDV, 2 de septiembre de 2017).

Si bien los procesos de socialización entre diferentes ayuda a la construcción de sentidos de respeto mutuo y favorecen la comprensión de la diversidad como condición humana, como bien lo demuestran estudios sobre la participación en el aula y la socialización (Bossaert et al, 2013), es indudable que la educación especial tiene un propósito importante para el desarrollo de capacidades en personas con limitaciones evidentes, a partir del acceso a apoyos requeridos, lo que menguaría el agravio moral de desconocer a cada persona en su diferencia, sin la pretensión de la homogenización de los cuerpos para su control social.

\section{Conclusiones}

La discapacidad es una condición que implica comprender la pluralidad humana, trascendiendo la concepción de modelos de cuerpos impuestos por las sociedades hegemónicas y excluyentes. Esta comprensión permite reconocer las limitaciones y las capacidades individuales, de tal manera que la asistencia contenga los apoyos necesarios para favorecer el desarrollo de la autonomía y el despliegue de un estilo de vida independiente. Suele ser tan destructiva la negación de la limitación como la concepción de que se posee una enfermedad, en ambos casos, se desconoce al ser humano en sí mismo con todas las implicaciones y aspectos que lo integran.

La mirada política de la discapacidad permite trascender la asistencia de beneficencia, hacia una asistencia desde el reconocimiento de las personas como fines en sí mismas, para deconstruir las concepciones que sobre sus cuerpos han dejado los modelos de prescindencia, médico-rehabilitador, incluso, social. La autonomía moral y la capacidad de configurar estilos de vida independientes cobran sentido, siempre que los procesos de socialización favorezcan relaciones de reconocimiento.

Aquí se gesta la posibilidad de que las personas con discapacidad se consideren a sí mismas como sujetos legítimos de derechos; en tanto humanos y en tanto diversos, podrán alcanzar mayores desarrollos de autonomía por la confianza en sí, el conocimiento de sus derechos jurídicos y el aprecio propio como sujetos valiosos en la sociedad, de allí que tomarán decisiones sobre su vida y sobre su contexto.

Los participantes narran las vivencias que permiten develar, que han atravesado las tres esferas de reconocimiento en los diversos grupos familiares, comunitarios y laborales, y que ello les ha permitido devenir en sujetos con autoconfianza, autorrespeto y autoestima considerados fines prácticos del reconocimiento, lo que ha favorecido el desarrollo de la autonomía y los modos de vida independiente. Este fue el primer hallazgo de la investigación.

El segundo hallazgo muestra la existencia de lo denominado como enemigos del reconocimiento en el contexto de la discapacidad, teniendo en cuenta que, en cada narración, si bien aparecen las experiencias favorables para el devenir de los sujetos como fines en sí mismos y valorados en sus diversidades, también 
se develan agravios morales. Se resalta que no hay relatos sobre agravios físicos, pero es evidente la vivencia de negación de derechos, que es uno de los agravios morales más frecuentes para las personas con discapacidad; además, con mayor frecuencia, se demuestran agravios morales referidos a la degradación social, que es contraria a la solidaridad y al reconocimiento de estas personas como sujetos diversos, con valor propio y capacidades importantes para la construcción colectiva del mundo, más allá de la visión de la productividad. 


\section{Referencias bibliográficas}

Arendt, H. (2005). La condición humana. Barcelona: Paidós Ibérica.

Berger, P. y Luckmann, T. (2001). La construcción social de la realidad. Buenos Aires: Amorrortu.

Bossaert, H., et al. (2013). "Social participation of students with special educational needs in mainstream seventh grade”. Procedia. Social and Behavioral Sciences, 93(21), pp. 1952-1956.

Buitrago-Echeverri, M. T. (2013). Discapacidades peregrinas. Construcciones sociales de la discapacidad en Colombia: Aportes para salud pública la desde una perspectiva crítica (Tesis doctoral, Universidad Nacional de Colombia). Recuperado de http://www.bdigital.unal.edu.co/10737/1/5598367.2013.pdf

Carbonell, G. J. (2019). "El movimiento de vida independiente en España". Revista Española de Discapacidad, 7 (II), pp. 201-214.

Coffey, A. y Atkinson, P. (2003). Encontrar el sentido a los datos cualitativos. Estrategias complementarias de investigación. Medellín: Universidad de Antioquia.

Díaz Alzate, M. V. y González Bedoya, D. M. (2015). "Experiencias de reconocimiento vividas por los niños en sus familias". Revista Colombiana de Ciencias Sociales, 6 (2), pp. 386-403.

Erikson, E. H. (1985). El ciclo vital completado. Buenos Aires: Paidós.

Fernández, A. y Vasco, E. (2012). "Dinámicas del reconocimiento en las narraciones de jóvenes con discapacidades”. Revista Latinoamericana de Ciencias Sociales, Niñez y Juventud, 10(1), pp. 467-479.

Galeano, M. E. (2004). Estrategias de investigación social cualitativa. El giro en la mirada. Medellín: La Carreta.

Hernández Ríos, M. I. (2015). "El concepto de discapacidad: De la enfermedad al enfoque de derechos". Revista CES Derecho, 6(2), pp. 46-59.

Honneth, A. (1992). "Integridad y desprecio. Motivos básicos de una concepción de la moral desde la teoría del reconocimiento". Isegoría, Revista de Filosofía Moral y Política, 5, pp. 78-92.

Honneth, A. (1996). "Reconocimiento y obligaciones morales". Revista Internacional de Filosofía Política RIFP, 8 , pp. 5-17.

Honneth, A. (1997). La lucha por el reconocimiento. Por una gramática moral de los conflictos sociales. Barcelona: Crítica Grijalbo.

Honneth, A. (2004). Recognition and Justice. Outline of a Plural Theory of Justice. Acta Sociologica, 47(49), pp. 351-364.

Honneth, A. (2009). Crítica del agravio moral. Patologías de la sociedad contemporánea. Buenos Aires: Fondo de Cultura Económica.

Honneth, A. (2010). Reconocimiento y menosprecio. Sobre la fundamentación normativa de una teoría social. Madrid: Katz.

Honneth, A. (2011). La sociedad del desprecio. Madrid: Trotta.

láñez-Domínguez, A. (2009). Prisioneros del cuerpo. La construcción social de la diversidad funcional. Madrid: Diversitas Ediciones. 
Maraña, J. J. (2004). Vida independiente. Nuevos modelos organizativos. Madrid: Asociación Iniciativas y Estudios Sociales.

Martínez Miguélez, M. (2004). Ciencia y arte en la investigación cualitativa. México D.C.: Trillas.

Mead, G. (1968). Espíritu, persona y sociedad, desde el punto de vista del conductismo social. Buenos Aires: Paidós.

Naciones Unidas (2006). Convención sobre los Derechos de las Personas con Discapacidad. Nueva York: ONU.

Nussbaum, M. (2012). Las fronteras de la justicia. Consideraciones sobre la exclusión. Barcelona: Paidós Ibérica.

Organización Mundial de la Salud (OMS) (2001). Clasificación Internacional del Funcionamiento, de la Discapacidad y de la Salud (CIF). Madrid: Grafo. Recuperado de http://apps.who.int/iris/bitstream/handle/10665/43360/9241545445_spa.pdf;jsessionid=CB381C783F3C4DEEBB9B9A58FAF29BA3?sequence=1

Ospina Ramírez, M. A. (2010). "Discapacidad y sociedad democrática”. Revista Derecho del Estado, 24, pp. 143164.

Palacios, A. y Romañach, J. (2006). El modelo de la diversidad. La bioética y los derechos humanos como herramientas para alcanzar la plena dignidad en la diversidad funcional. Madrid: Diversitas Ediciones.

Pérez, M. E. y Chhabra, G. (2019). "Modelos teóricos de discapacidad: un seguimiento del desarrollo histórico del concepto de discapacidad en las últimas cinco décadas". Revista Española de Discapacidad, 7(I), pp. 7-27.

Ruiz Silva, A. (2004). "Texto, testimonio y metatexto. El análisis de contenido en la investigación en educación”. En A. Jiménez Becerra y A. Torres Castillo, A. (comp.), La práctica investigativa en ciencias sociales (pp. 44-59). Bogotá: Universidad Pedagógica Nacional.

Strauss, A. y Corbin, J. (2002). Bases de la investigación cualitativa. Técnicas y procedimientos para desarrollar teoría fundamentada. Medellín: Universidad de Antioquia.

Thompson, J. R. et al. (2004). SIS. Escala de Intensidad de Apoyos. Madrid: TEA Ediciones.

Valles, M. (2002). "Entrevistas cualitativas”. Cuadernos Metodológicos, 32, pp. 1-195. 